\title{
Enhanced thermal and mechanical properties of poly(trimethylene terephthalate-block-poly(tetramethylene oxide) segmented copolymer based hybrid nanocomposites prepared by in situ polymerization via synergy effect between SWCNTs and graphene nanoplatelets
}

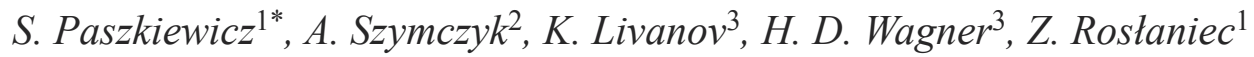 \\ ${ }^{1}$ West Pomeranian University of Technology, Institute of Material Science and Engineering, Piastow Av. 19, PL-70310 \\ Szczecin, Poland \\ ${ }^{2}$ West Pomeranian University of Technology, Institute of Physics, Piastow Av. 19, PL-70310 Szczecin, Poland \\ ${ }^{3}$ Department of Materials and Interfaces, Weizmann Institute of Science, 76100 Rehovot, Israel
}

Received 9 October 2014; accepted in revised form24 December 2014

\begin{abstract}
Graphene nanoplatelets/single walled carbon nanotubes/poly(trimethylene terephthalate-block-poly(tetramethylene oxide) segmented copolymer (GNP/SWCNT/PTT-PTMO) hybrid nanocomposites were synthesized via in situ polymerization. A remarkable synergistic effect between GNPs and SWCNTs on improving thermal and mechanical properties of nanocomposites based on segmented block copolymers was observed. Heterogeneous structure of the PTT-PTMO allowed for a better and more uniform distribution of both types of nanoparticles and stabilized the structure in question. This enabled us to observe a so-called 'synergistic effect', caused by the use of mixture of carbon nanotubes and graphene nanopletelets, on the enhancement of thermal and mechanical properties of the obtained polymer. In order to ascertain the influence of mentioned carbon nanostructures on the nano-phase-separated structure of the synthesized PTT-PTMO block copolymers, differential scanning calorimetric (DSC) and dynamic mechanical thermoanalysis (DMTA) measurements were performed. Scanning electron microscopic (SEM) and transmission electron microscopic (TEM) images of the PTTPTMO nanocomposites displayed that hybrid nanofillers exhibited better distribution and compatibility than SWCNTs and GNPs did individually. The tensile modulus of $0.5 \mathrm{SWCNT} / 0.1 \mathrm{GNP} / \mathrm{PTT}-\mathrm{PTMO}$ composites was $68 \%$ higher than that of the PTT-PTMO alone, compared to only a 10 and $28 \%$ increase in tensile modulus for $0.3 \mathrm{GNP} / \mathrm{PTT}-\mathrm{PTMO}$ and 0.3SWCNT/PTT-PTMO composites respectively (the highest concentration when single nanofiller was added).
\end{abstract}

Keywords: nanocomposites, nanomaterials, mechanical properties, thermal properties, material testing

\section{Introduction}

The field of nanocomposite and polymer nanocomposite research is currently one of the most rapidly developing domains of cognitive work and applied engineering. The materials in question are essentially complex systems with two or more phases (continuous and dispersed) with an explicit separation surface. The studies on interfacial interactions at the nano level which affect the composite's properties are among the most important subjects of research and development in the field of material science today $[1,2]$.

Up to and including now, nanocomposites enriched by an addition of carbon nanotubes have sparked great interest among scientists and innovative research groups [3]. Same has been the case for

\footnotetext{
${ }^{*}$ Corresponding author, e-mail: spaszkiewicz@zut.edu.pl (C) BME-PT
} 
graphene, an allotropic form of carbon discovered in 2004, existence of which was earlier only predicted by complex theoretic models. Graphene itself is a one atom thick, two-dimensional (2D) sheet composed of $\mathrm{sp}^{2}$ carbon atoms arranged in a honeycomb lattice [4] with a carbon-carbon bond length of $0.142 \mathrm{~nm}[5,6]$. It has proven to have a variety of exceptional intrinsic characteristics among which high electron mobility at room temperature $\left(250000 \mathrm{~cm}^{2} /(\mathrm{V} \cdot \mathrm{s})\right)[7,8]$, (where, unlike in the case of CNTs, chirality does not impede it's electrical conductivity), exceptional thermal conductivity $(5000 \mathrm{~W} /(\mathrm{m} \cdot \mathrm{K}))$ [9], and superior mechanical properties with Young's modulus of $\sim 1 \mathrm{TPa}$ and ultimate strength of $130 \mathrm{GPa}$ [8] can be distinguished. Aforementioned characteristics, along with an extremely high surface area (theoretical limit: $2630 \mathrm{~m}^{2} / \mathrm{g}$ ) and gas impermeability [10], demonstrate graphene's unique potential for improving electrical, mechanical, thermal and gas barrier properties of polymers $[11,12]$. Taking all above into consideration, a simultaneous introduction of two types of carbon nanofillers such as graphene (a plate filler-2D) and CNTs (a fibrous/linear filler - 1D) may seem desirable from the standpoint of their influence on electrical and mechanical qualities.

A controlled distribution of both nanoplatelets and linear nanoparticles throughout a polymer matrix may result in obtaining specified physical characteristics, most important being the enhancement of mechanical properties and obtaining electrically conductive materials. All at a very low total concentration of both nanofillers. This claim is substantiated by several cases i.e. thin polymer films have been fabricated from a mixture of single walled carbon nanotubes (SWCNTs) and multi walled carbon nanotubes $[13,14]$ and from a mixture of SWCNTs and carbon fibers [14]. In each mentioned case the examined samples displayed an array of significantly improved properties. Furthermore, another recent research has looked at the mixtures of oxidized nanotubes and graphene oxide [15]. Finally, Khan et al. [16] prepared hybrid films comprised of single walled carbon nanotubes and nano-graphite. It has been demonstrated that these hybrids displayed mechanical and electrical properties that were superior to those of either nanotube-only or graphene-only films.

Latest literature on the subject indicates that it was not yet possible to fully utilize the potential of apply- ing a mixture of carbon nanotubes and graphene to observe a so-called 'synergistic effect' which in theory should result in a significant enhancement of properties of obtained materials. Indeed, a remarkable synergistic effect between multi-graphene platelets (MGPs) and multi walled carbon nanotubes (MWCNTs) causing an improvement of mechanical properties and thermal conductivity in epoxy composites has been studied [17]. However, obtaining similar results for thermoplastic polymer composites remains an unresolved and thus a noteworthy subject, hence it is widely undertaken by many research groups. Therefore, the addition of carbon nanoparticles to segmented block copolymers, composed of both flexible and rigid segments, seems particularly interesting as they demonstrate a behaviour similar to that of thermoplastic elastomers. Due to their excellent mechanical qualities, like strength and elastic properties in a wide temperature range, multiblock poly(ether-esters) (PEEs) have been intensely studied $[18,19]$. A recent study on a novel family of polyester thermoplastic elastomers based on poly(trimethylene terephthalate) (PTT) has been conducted [20,21]. The influence of organoclay [22], carbon nanotubes [23] and graphene oxide [24] on the structure and physical properties of PTT-PTMO copolymers has been previously described. This type of segmented block copolymers, based on poly(trimethylene terephthalate) (PTT) as rigid segments and polyether (PTMO) as flexible segments, displays a variety of remarkable qualities such as low glass transition temperature $\left(T_{\mathrm{g}}\right)$, high melting temperature of rigid phase $\left(T_{\mathrm{m}}\right)$ and a temperature independent rubbery plateau. An addition of carbon nanoparticles in mentioned block copolymers may influence the phase separation thus changing the elastic properties of the used polymer matrix. It is anticipated that the heterogeneity of the PTTPTMO structure will allow for its stabilization and a better and more uniform dispersion of nanoparticles. Although the phase size of polyester thermoplastic elastomers is greater than the size of nanoparticles, it is believed that the multiphase structure of block copolymers will significantly improve both thermal and mechanical characteristics. Moreover, it will allow to observe a synergistic effect between carbon nanotubes and graphene nanoplatelets on properties enhancement

This work is a part of a wider project with an objective to fabricate electrically conductive polymer 
nanocomposites containing carbon nanoparticles [25]. In this study, PTT- PTMO based nanocomposites with hybrid fillers comprising of SWCNTs and GNPs were developed in order to enhance thermal stability and mechanical characteristics. The thermal and mechanical properties of the obtained nanocomposites were thoroughly evaluated and favourably compared with those of neat block copolymer and those containing either SWCNTs or GNPs alone.

\section{Experimental section}

\subsection{Materials}

The single walled carbon nanotubes KNT 95 (SWCNTs) were purchased from Grafen Chemical Industries (Grafen Co., located in Ankara, Turtkey) a leader in the field of fabrication and application of carbon nanomaterials. According to manufacturers' data: diameter: $<2 \mathrm{~nm}$, electrical conductivity $(E C)$ : $>100 \mathrm{~S} / \mathrm{cm}$, length: 5-30 $\mu \mathrm{m}$, purity: $>95 \%$, surface area: $380 \mathrm{~m}^{2} / \mathrm{g}$. Graphene nanoplatelets $(<1 \mathrm{~nm})$ was purchased from ANGSTRON Materials (Dayton, Ohio, USA), it was bought in the form of a powder with less than three graphene layers and $x-y$ dimensions of up to $10 \mu \mathrm{m}$. The carbon content is $\sim 97.0 \%$, and the oxygen content is $\sim 2.10 \%$. Graphene nanoplatelets have a specific surface area of 400 $800 \mathrm{~m}^{2} / \mathrm{g}$ and a true density of $\leq 2.20 \mathrm{~g} / \mathrm{cm}^{3}$.

The following materials were used in the course of fabrication of the PTT-PTMO block copolymer and PTT-PTMO/carbon nanoparticles nanocomposites: dimethyl terephtalate (DMT, Sigma-Aldrich) and poly(tetramethylene oxide) glycol with molecular mass of $1000 \mathrm{~g} / \mathrm{mol}$ (PTMG, Terathane 1000, DuPont, USA) were used as received. 1,3-propanenediol (PDO, Sigma-Aldrich) was distilled before use. Tetrabutyl orthotitaniate (TBT, Fluka) was used as catalyst in transesterification and polycondensation. Irganox 1010 (Ciba-Geigy, Switzerland) was used as antioxidant.

\subsection{Synthesis of PTT-PTMO based nanocomposites}

Poly(trimethylene terephthate-block-poly(tetramethylene oxide) (PTT-PTMO) segmented block copolymer based nanocomposites with carbon nanotubes and/or graphene nanoplatelets were prepared by in situ polymerization according to a procedure described earlier for nanocomposites with MMT [22] and GO [24]. The PTT-PTMO block copolymer with the content of $50 \mathrm{wt} \%$ of PTT rigid segments and $50 \mathrm{wt} \%$ of PTMO soft segments was used as nanocomposite polymer matrix. The process of in situ polymerization was preceded by a stage of nanofillers dispersion. For that purpose a specified amount of graphene nanoplatelets was mixed in a liquid substrate (1,3-propylene glycol) during a $30 \mathrm{~min}$ period, alternately using a high speed stirrer and vibrations of an ultrasound stirrer. Subsequently, for the purpose of greater separation of graphene sheets an ultrasonic washer was used for a period of 8 hours. To a so prepared mixture of graphene nanoplatelets, in the case of hybrid nanocomposites, a specified amount of carbon nanotubes was added. However, due to the high fragility of nanotubes only an ultrahigh speed stirrer high (Ultra-Turrax ${ }^{\circledR}$ T25) and ultrasonic homogenizer (Sonopuls HD 2200, Bandelin) were used in order to prepare their dispersion in an appropriate manner. A ready dispersion was then put into a polycondensation reactor (Autoclave Engineers, Pennsylvania, USA), preheated to $80^{\circ} \mathrm{C}$, along with dimethyl terephtalate (DMT) and a first portion of catalyst. First stage of the reaction, the transesterification process, was performed in a $160-185^{\circ} \mathrm{C}$ temperature range. The course of the reaction was controlled by observing the secretion of its by-product - methanol. When its amount reached about $90 \%$ of the quantity computed by means of stoichiometric calculations, the second stage of synthesis, being the polycondensation process, was commenced. For this purpose PTMO mixed with thermal stabilizer (Irganox 1098), along with the second portion of catalyst were inserted. The temperature of the reaction was then gradually increased up to $250^{\circ} \mathrm{C}$. However, unlike the first stage which was performed under atmospheric pressure, the polycondensation stage was carried out under reduced pressure $(\sim 20 \mathrm{~Pa})$. In this case, the course of the reaction was monitored by observing the stirrer's torque. When the polymer/nanocomposite melt reached a high molecular mass, it was then extruded in the form of a thin wire. It was subsequently granulated and subjected to injection moulding procedure.

\subsection{Preparation of test samples}

The dumbbell shaped samples for density, DMTA, SEM and tensile tests were obtained using a Boy 15 (Dr BOY GmbH\&Co., Germany) injection moulding machine with parameters as follows: injection pressure $55 \mathrm{MPa}$, melt temperature $225^{\circ} \mathrm{C}$, mould temperature $30^{\circ} \mathrm{C}$, holding down pressure of $20 \mathrm{MPa}$ 
for $15 \mathrm{~s}$ and cooling time $10 \mathrm{~s}$. Prior to performing the above mentioned measurements, the dumbbell shaped samples were annealed at $80^{\circ} \mathrm{C}$ for a period of $2 \mathrm{~h}$.

\subsection{Methods of characterization}

The intrinsic viscosity $[\eta]$ of the obtained samples was determined using a phenol/1,1,2,2-tetrachloroethane mixture $\left(60 / 40\right.$ by weight) at $30^{\circ} \mathrm{C}$. The concentration of polymer in the solvent amounted to $0.5 \mathrm{dL} / \mathrm{g}$. The measurement was performed using a capillary viscometer Ubbelohde (type Ic, $K=$ 0.03294). In order to remove the influence of the nanofiller on the measured values, a procedure described earlier in [22, 25-27] was used.

Density measurement $(d)$ was performed using a hydrostatic scales (Radwag WPE 600C, Poland), calibrated using working standards of known density. The melt viscosity of the samples was measured using ARES rheometer (Rheometric Scientific Inc., USA). The measurement was performed at $220^{\circ} \mathrm{C}$ in frequency range $0.1-50 \mathrm{~Hz}$, in a parallel-plate fixture (diameter $=25 \mathrm{~mm}$ ) with a gap distance of $2 \mathrm{~mm}$.

The structure of nanocomposites was observed using scanning electron microscopes (SEM, JEOL JSM 6100; SEM ULTRA-55 Zeiss and SEM SUPRA-55 VP Zeiss). Prior to testing, the samples were cryofractured in liquid nitrogen, and then vacuum coated with a thin gold film. SEM images were collected with an In-Lens detector at an acceleration voltage of $5 \mathrm{kV}$ and working distance of 5-6 mm. Transmission electron microscopic (TEM) analysis was carried out by a PHILLIPS CM 120 Electron Microscope using an acceleration voltage of $80 \mathrm{kV}$. The specimens used in TEM examination (thickness about $100-150 \mathrm{~nm}$ ) were obtained/cut from the central part of the dumbbell shaped sample using Reichert Ultracut $\mathrm{R}$ ultramicrotome with a diamond knife. The procedure was carried out only after the sample had been immersed in liquid nitrogen. The direction of the cut was perpendicular to the direction of flow in the mould.

Thermal and thermo-oxidative stability of investigated polymer nanocomposites were evaluated by thermogravimetry (TGA 92-16.18 Setaram) using the system to measure the simultaneous TG-DSC. Measurements were carried out in inert atmosphere (argon) an oxidizing atmosphere i.e. dry, synthetic air $\left(\mathrm{N}_{2}: \mathrm{O}_{2}=80: 20 \mathrm{vol} \%\right)$. The study was conducted at a heating rate of $10^{\circ} \mathrm{C} / \mathrm{min}$ in the temperature range $20-700^{\circ} \mathrm{C}$. Measurements were conducted in accordance with the principles contained in the PN-EN ISO 11358:2004.

The dynamic mechanical thermal (DMTA) measurements were performed using a Polymer Laboratories MK II apparatus working in a bending mode at a frequency of $1 \mathrm{~Hz}$ in a temperature range from $-100^{\circ} \mathrm{C}$ to polymer melt temperature and heating rate of $3^{\circ} \mathrm{C} / \mathrm{min}$. Dynamic mechanical thermal analysis comprised of measurements of $E^{\prime}$ constituents (storage modulus) and $E^{\prime \prime}$ (loss modulus) complex elastic modulus and mechanical loss factor $\tan \delta=$ $\left(E^{\prime \prime} / E^{\prime}\right)$. It is one of the relaxation methods, which allows to ascertain the structure and phase transformation of the researched materials. The examined sample is subjected to a sinusoidal alternating causing its' deformation, the properties are determined on the basis of modulus changes and the ability of attenuation as a function of temperature and frequency of load changes. Temperatures of $\alpha$-relaxation $\left(T_{\alpha}\right)$ and $\beta$-relaxation $\left(T_{\beta}\right)$ were designated from the maximum of the loss modulus change curve $\left(E^{\prime \prime}\right)$ and the loss factor tangent $(\tan \delta)$ of temperature curve.

Differential scanning calorimetry (DSC) measurements were carried out using a TA Q100 instrument under a nitrogen atmosphere in a temperature range from -100 to $250^{\circ} \mathrm{C}$ at the heating and cooling rate of $10^{\circ} \mathrm{C} / \mathrm{min}$ and sample weight of $10 \pm 0.2 \mathrm{mg}$. Each DSC testing cycle consisted of heating, cooling and repeating of the scans. The first cooling and second heating scans were used to determine the melting and crystallization peaks. The heat of fusion has been determined by integration of the normalized area of melting endotherm. The glass transition temperature $\left(T_{\mathrm{g}}\right)$ of the polymer samples was taken as the midpoint of the change in heat capacity. The degree of crystallinity of the sample $\left(X_{\mathrm{c}}\right)$ was calculated using Equation (1):

$X_{\mathrm{c}}[\%]=\frac{\Delta H_{\mathrm{m}}}{\Delta H_{\mathrm{m}}^{0}}$

where $\Delta H_{\mathrm{m}}^{0}$ is the enthalpy change of melting for a $100 \%$ crystalline sample, for PTT equals to $146 \mathrm{~J} / \mathrm{g}$ [28] and $\Delta H_{\mathrm{m}}$ is derived from melting peak area on DSC thermogram.

Tensile measurements were performed on an Instron 5566 universal tensile testing frame, equipped with a $5 \mathrm{kN}$ Instron load cell, a contact optical long travel extensometer and the Bluehill 2 software. Pneu- 
matic $1 \mathrm{kN}$ Instron side action grips were used to clamp the test samples. The measurements were carried out at room temperature using a cross-head speed of $100 \mathrm{~mm} / \mathrm{min}$ and a grip distance of $20 \mathrm{~mm}$. The tensile properties, being the Young's modulus, yield stress and yield strain, stress and elongation at break, were determined using injection moulded dumbbell-shaped bars (ISO 37 type 3). The results for each polymer are based on data collected from 6 samples (per polymer), for which the mean values and standard deviations were calculated. Moreover, the tensile deformation recovery properties of the samples were investigated. The dumbbell sample was stretched to a predetermined strain at a speed of $100 \mathrm{~mm} / \mathrm{min}$ at room temperature, and then the imposed strength was released. The residual strain was then measured to calculate the deformation recovery rate. The predetermined strain of $100 \%$ was utilized in our testing.

\section{Results and discussion}

\subsection{Rheological properties}

A series of hybrid nanocomposites based on multiblock poly(ether-ester) copolymer with PTT as a rigid segment were synthesized via in situ polymerization. The values of intrinsic viscosity, melt viscosity and density (Table 1) are dependent on the sample composition. The measured values of intrinsic viscosity for PTT-PTMO copolymer $(1.325 \mathrm{dL} / \mathrm{g})$ and PTT-PTMO hybrid nanocomposites (1.2991.322) $[\mathrm{dL} / \mathrm{g}]$ (for three hybrids with increasing content of SWCNTs) suggest that molecular weights of studied polymers should be reasonably high. The value of $[\eta]$ decreased slightly along with addition of nanofillers. However, an increase was observed together with a rise in number of SWCNTs and graphene platelets.

It can clearly be seen that the addition of SWCNTs strongly affected the melt viscosity of PTT-PTMO

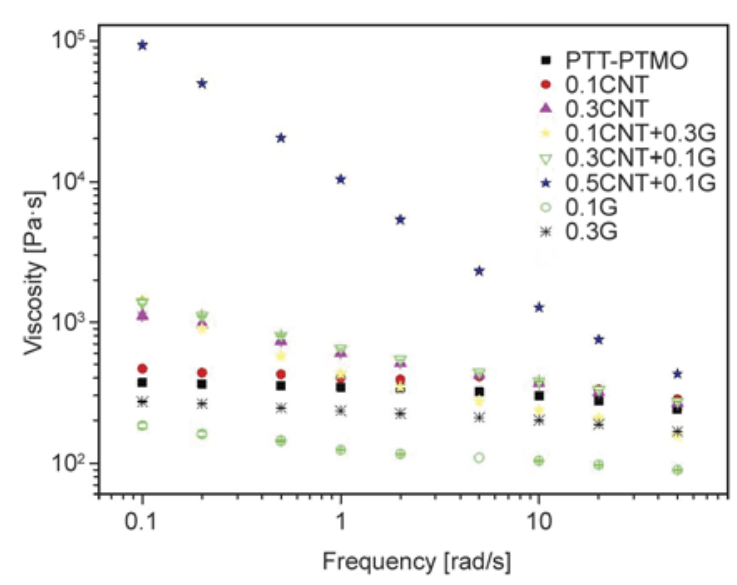

Figure 1. Melt viscosity versus frequency for neat PTTPTMO and PTT-PTMO/SWCNTs+GNPs nanocomposites at temperature of $220^{\circ} \mathrm{C}$

nanocomposites (Figure 1). On the other hand however, no such behaviour was observed when graphene nanoplatelets were added to the polymer (Table 1). A much stronger effect of the addition of carbon nanotubes on the viscosity of the composition was observed when compared to the hybrid nanocomposite PTT-PTMO/0.1 SWCNTs +0.3 GNPs with the same amount of SWCNTs $(0.1 \mathrm{wt} \%)$. Same was observed for the nanocomposite with $0.3 \mathrm{wt} \%$ of SWCNTs. It can therefore be concluded, that in case of PTT-PTMO hybrid nanocomposites, the mixture of both nanofillers affected the polymer's melt viscosity much stronger than either graphene or nanotubes alone (Figure 1). A similar effect was noticed in the case of nanocomposites based on polycarbonate with carbon black/carbon nanotube systems [29]. It has also been observed that an increase in pure MWCNT/CB content leads to respectively highest/lowest increase rate of melt viscosity. Additionally, the melt viscosity value for composites with mixed filler systems were in between those of pure/singe fillers. In the case described herein, addition of carbon nanoparticles with a $0.5 \mathrm{wt} \%$ nanotube content caused a substantial increase in viscos-

Table 1. Intrinsic viscosity, density and melt viscosity of PTT-PTMO/SWCNTs+GNPs nanocomposites

\begin{tabular}{|l|c|c|c|c|}
\multicolumn{1}{|c|}{ Sample } & $\begin{array}{c}\text { Nanofiller } \\
{[\mathbf{w t} \mathbf{m}]}\end{array}$ & $\begin{array}{c}{[\mathbf{q}]} \\
{[\mathbf{d L} / \mathbf{g}]}\end{array}$ & $\begin{array}{c}\mathbf{d} \\
{\left[\mathbf{g} / \mathbf{c m}^{\mathbf{3}}\right]}\end{array}$ & $\begin{array}{c}\text { Melt viscosity }^{\mathbf{M}} \\
{[\text { Pa·s] }}\end{array}$ \\
\hline PTT-PTMO & 0 & 1.325 & 1.174 & $340.02 \pm 0.76$ \\
\hline PTT-PTMO/0.1 G & 0.1 & 1.315 & 1.174 & $124.38 \pm 0.93$ \\
\hline PTT-PTMO/0.3 G & 0.3 & 1.327 & 1.175 & $232.66 \pm 0.84$ \\
\hline PTT-PTMO/0.1 SWCNT+0.3 G & $0.1+0.3$ & 1.299 & 1.173 & $428.76 \pm 1.22$ \\
\hline PTT-PTMO/0.3 SWCNT+0.1 G & $0.3+0.1$ & 1.319 & 1.177 & $648.65 \pm 1.31$ \\
\hline PTT-PTMO/0.5 SWCNT+0.1 G & $0.5+0.1$ & 1.322 & 1.176 & $10409.07 \pm 18.84$ \\
\hline PTT-PTMO/0.1 SWCNT & 0.1 & 1.325 & 1.181 & $398.52 \pm 1.08$ \\
\hline PTT-PTMO/0.3 SWCNT & 0.3 & 1.324 & 1.176 & $602.11 \pm 1.60$ \\
\hline
\end{tabular}

$[\eta]$ - intrinsic viscosity; $d$ - density; $\eta$ - melt viscosity measured at $220^{\circ} \mathrm{C}$ at $1 \mathrm{~Hz}$ 
ity value (over a 30 time increase relative to neat copolymer). The aforementioned concentration of nanofillers should be considered as a critical value for the used in situ polymerization method. When surpassed, extrusion of polymer from the reactor would be impossible. This indicates that despite an enhancement of mechanical and thermal qualities which is caused by the synergistic effect between SWCNTs and GNPs, the system comprised of 0.5 SWCNTs +0.1 GNPs provides the highest melt viscosity values along with the greatest improvement in tensile properties and thermal stability. However, such a high rise in viscosity may prove unsuitable for processing purposes.

The density of PTT-PTMO hybrid nanocomposites has increased slightly with the addition of nanofillers. Obtained density values depend strongly on the crystallite content as well as on the content of the nanofiller in the polymer matrix. As the obtained hybrid nanocomposites displayed density values comparable to those of nanotubes only, it may be concluded that an addition of nanotubes alone has a greater impact of the density.

\subsection{Morphology}

Single walled carbon nanotubes and graphene nanoplatelets used in this study were characterized using scanning electron microscopy (SEM), which provided visual information on the ratio of carbon nanofiller to carbonaceous impurities (Figure 2a and 2b). In case of SWCNTs it was presumed that fiber-like areas correspond to bundled SWCNTs, while the other particles and lumps correspond to carbonaceous impurities. Figure $2 \mathrm{~b}$ displays the photomicrograph of the loose structures of graphene nanoplatelets in sequentially larger magnifications. Obviously, in the case of graphene nanoplatelets loose structures containing multi-pores can be observed. This micrograph also suggests the thickness of the graphene platelet layer is less than $100 \mathrm{~nm}$.

As mentioned in the introduction, the natural tendency of carbon nanostructures to agglomerate (due to van der Waals interactions) is the underlying reason for difficulties in preparation of composites based on thermoplastic matrices, which can be characterized by a high degree of homogeneity of the nanophase. Effectiveness of using the in situ polycondensation method, preceded by a dispersion of the carbon nanotubes and/or graphene nanoplatelets in a substrate, can be assessed qualitatively by means of structure analysis using microscopic techniques. Taking into consideration the development of methods of in situ polymer composite preparation, it was assumed that dispersing SWCNTs/GNPs in one of the substrates by means of mechanical forces and vibrations at the ultrasonic frequency, and then carrying out the synthesis of the polymer matrix in the presence of the nanoparticles, would allow to obtain uniform distribution in the mass. The uniform distribution itself is owed to intercalation of growing polymer chains between nanotube bundles and graphene nanoplatelet, causing them to separate even further. One of the main problems encountered during dispersion of CNTs, limiting the effective use of carbon nanotubes as nanofillers in a polymer matrix, is the strong inter-tube attraction between CNTs. Many approaches to overcome this issue have been made, however they mostly result in an excessive modification or even damage to the unique morphology of CNTs. An efficient alternative allowing to tailor the polymer/CNT interface and at the same time preserving the integrity of the tubes is mixing them together with a different shaped nanofiller (i.e. platelike shape). In the discussed study, it was assumed that application of graphene nanoplatelets might be a suitable solution. A uniform distribution of SWCNTs on graphene's surface should cause a synergistic effect and thus enable them to act together as stronger reinforcing agent for the polymer, resulting in a substantial enhancement of thermal and mechanical properties (Figure 2c-2g). The evenly spaced bright dots and lines are attributed to the ends and edges of the broken SWCNTs/GNPs covered in PTT-PTMO. They are clearly visible due to their high electrical conductivity. A good connection between graphene nanoplatelets, contained in the SWCNTs and GNPs mixture, along with a good compatibility with the polymer matrix were due to residual oxide groups on GNPs' surface [25]. The dual-filler strategy appears to yield a more efficient dispersion of SWCNTs+GNPs/PTT-PTMO. The SEM images of the PTT-PTMO/0.3 SWCNT+0.1 GNPs are shown in Figure 2e-2g. Both the SWCNTs and GNPS show fairly good distribution which can be seen in Figure $2 \mathrm{e}$ and $2 \mathrm{~g}$. The graphene nanosheets in Figure 2e appear to show better dispersion than those previously observed when only graphene was added to copolymer matrix (incompletely exfoliated structure of nanocomposites based on PTT-PTMO with 

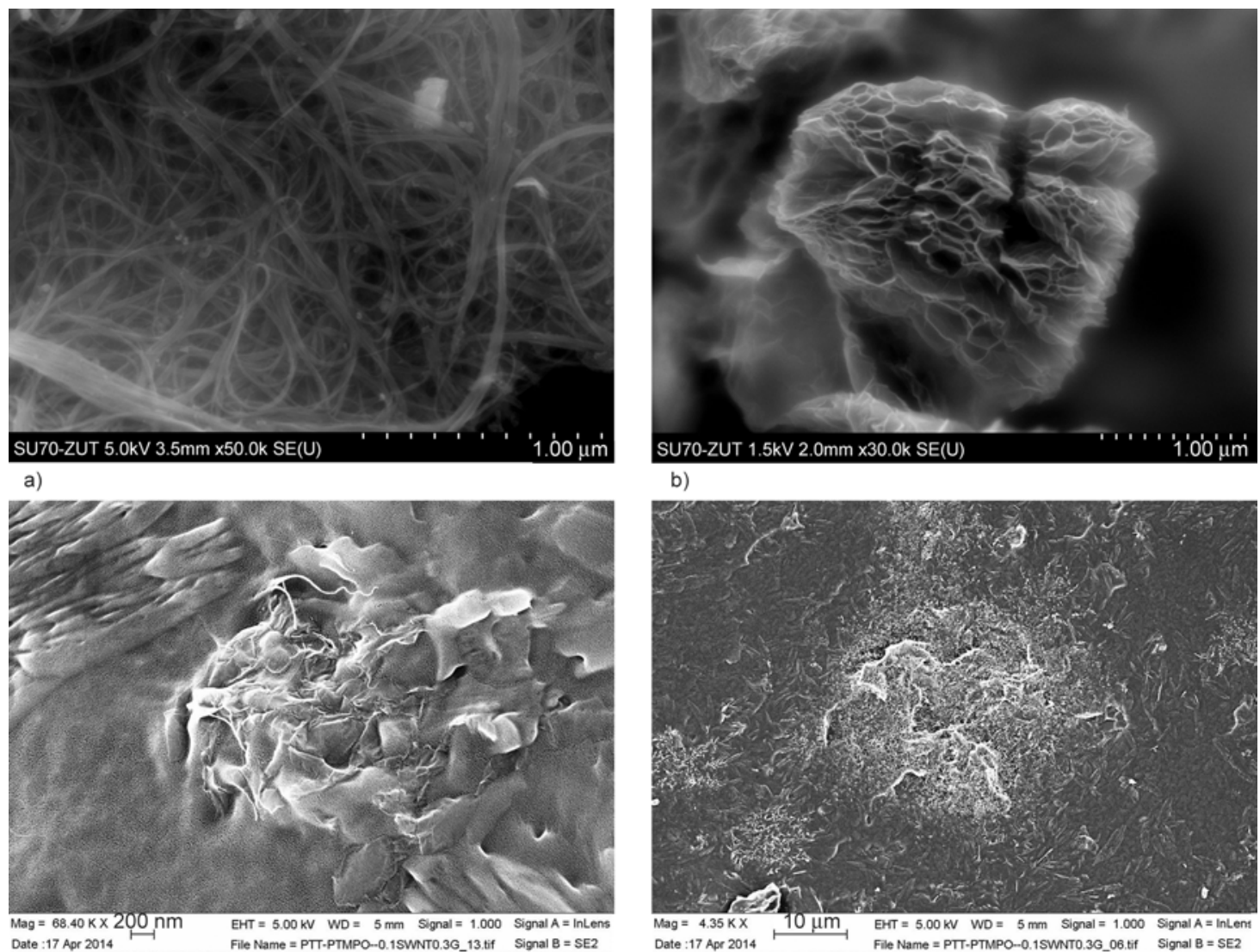

b)

c)

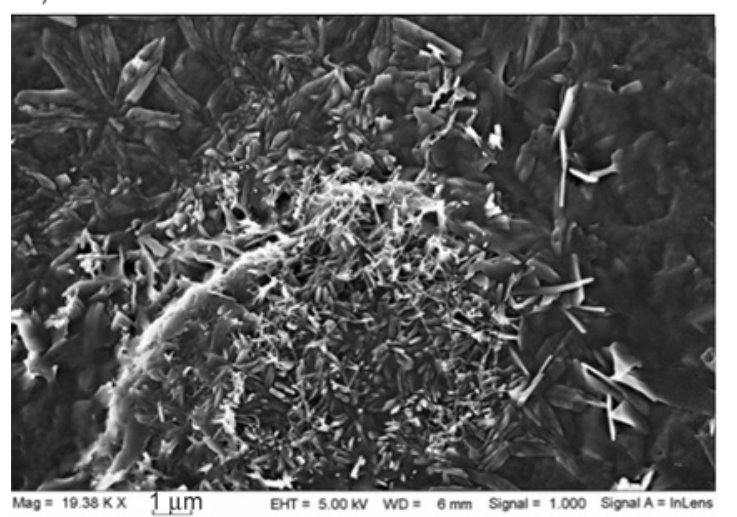

e)

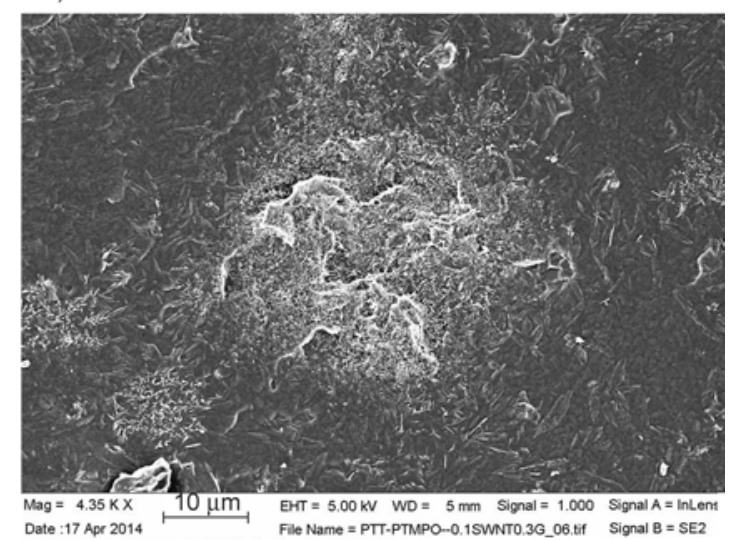

d)
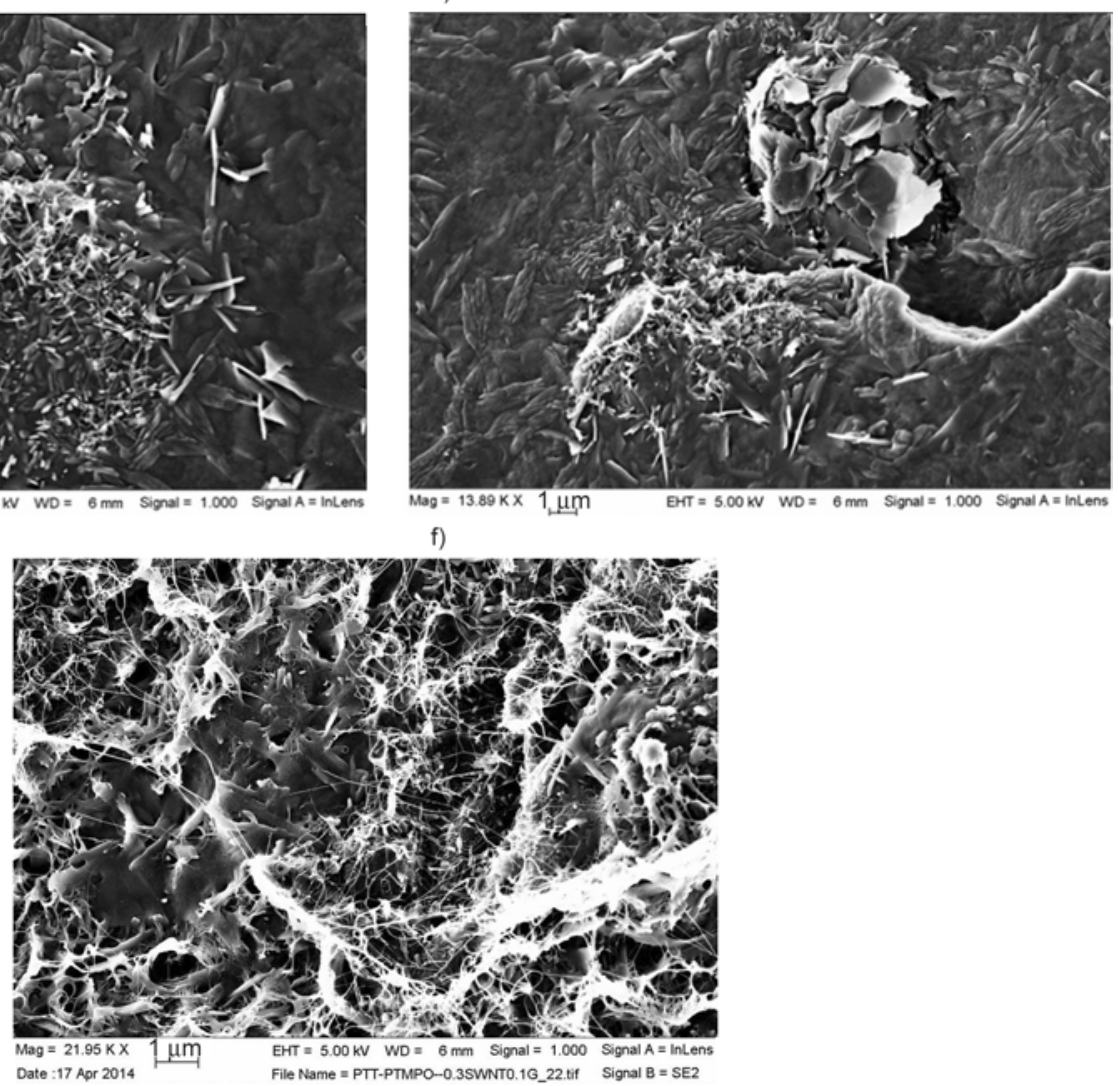

g)

Figure 2. (a) and (b) SEM micrographs of SWCNTs and GNPs as received; (c) and (d) SEM micrographs of PTTPTMO/0.1 SWCNTs+0.3 GNPs hybrid nanocomposites; (e)-(g) SEM micrographs of PTT-PTMO/0.3 SWCNT+ 0.1 GNPs hybrid nanocomposites 
GNP observed in TEM micrographs, Figure 3a, 3b). A detailed study on the influence of GNPs and GO on the morphology of PTT-PTMO matrix was previously described in [25] and [24] respectively. In that particular case this indicates that an addition of

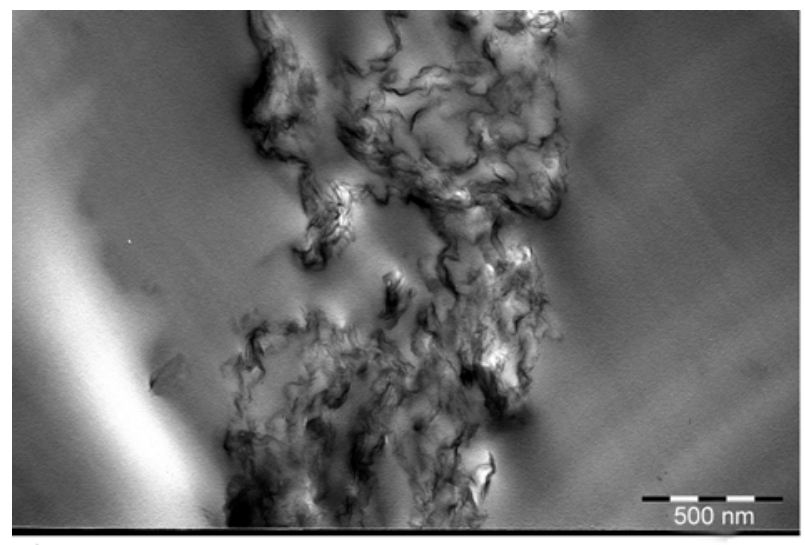

a)

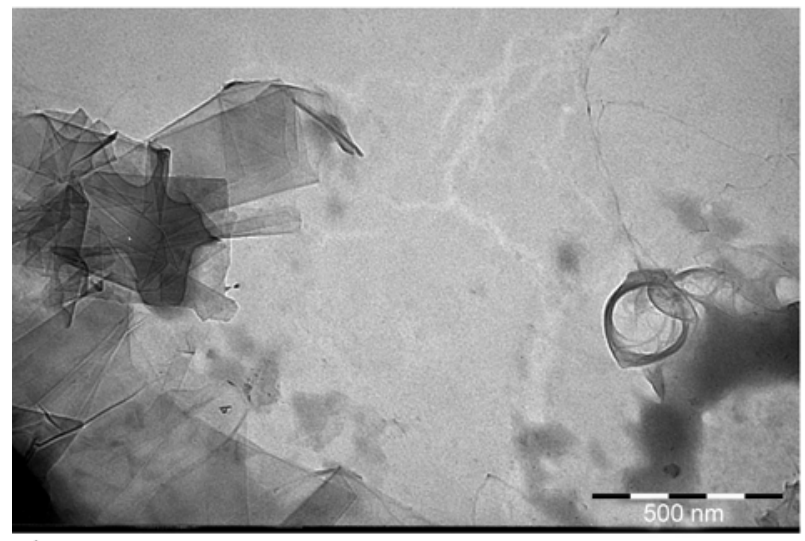

c)

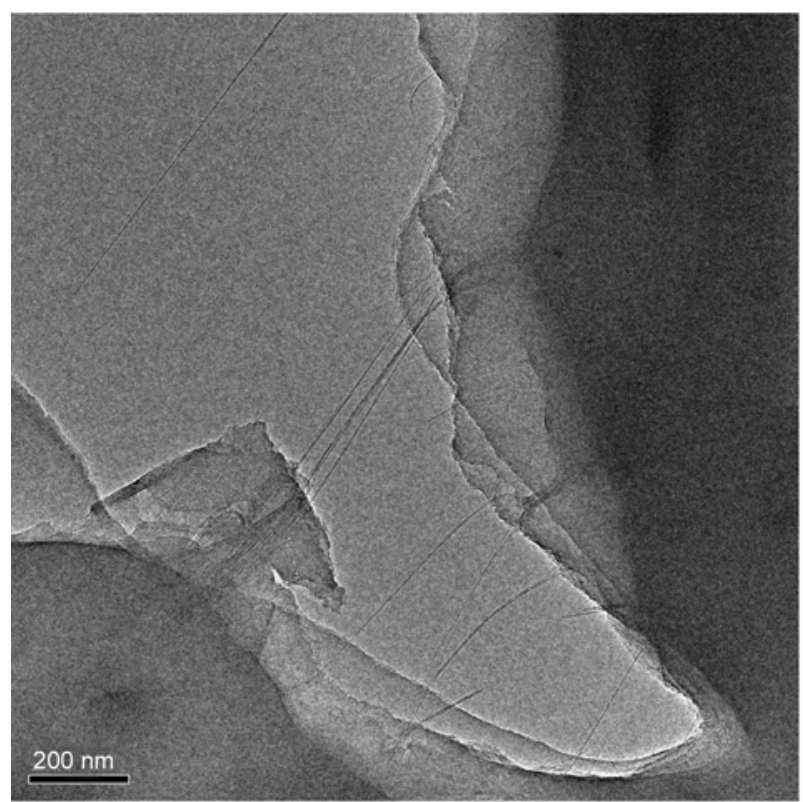

e)

Figure 3. (a)-(d) TEM micrographs of PTT-PTMO/GNPs nanocomposites with: (a) 0.1 GNPs, 75 000×, (b) 0.3 GNPs, $20000 \times$, (c) and (d) TEM micrographs of PTT-PTMO/0.1 SWCNT+0.3 GNPs nanocomposites at (c) $100000 \times$ and (d) $150000 \times$; (e) and (f) TEM micrographs of PTT-PTMO/0.3 SWCNT+0.1 GNPs nanocomposites (e) $150000 \times$ and (f) $175000 \times$
SWCNTs improves the dispersion of graphene nanoplatelets, which agrees with the tensile results which exhibit an improvement in mechanical qualities. However, because of the van der Waals interactions the graphene sheets tend to form irreversible agglom-

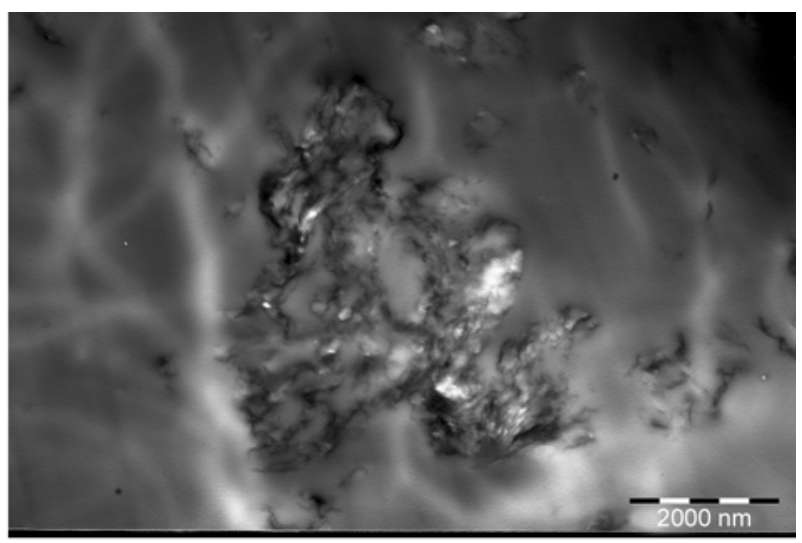

b)

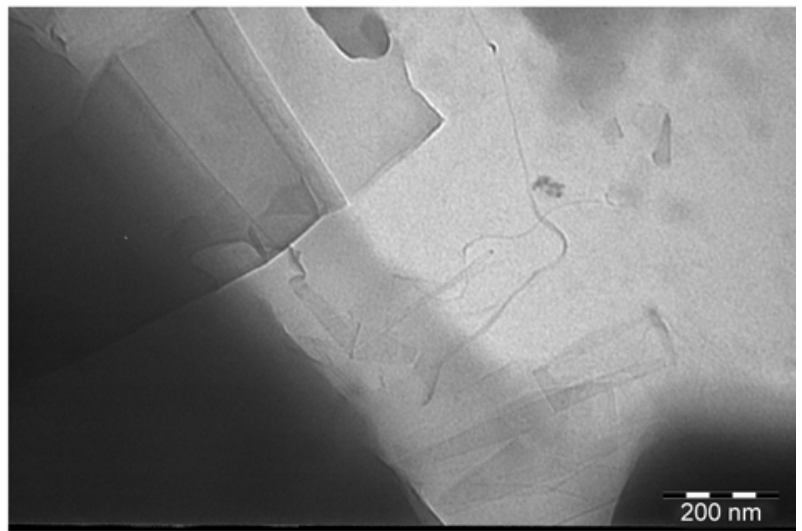

d)

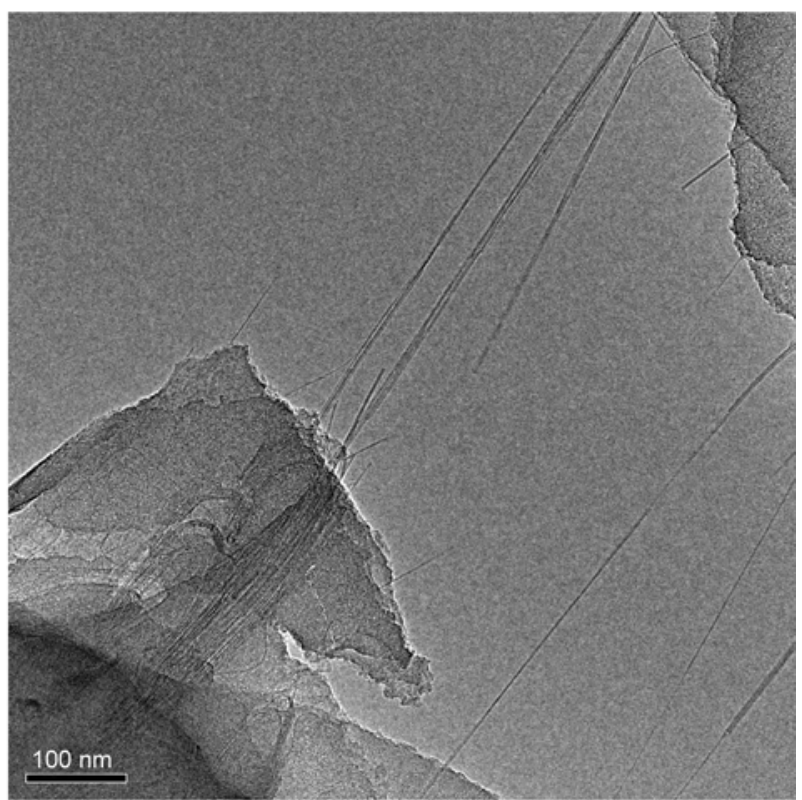

f) 
erates and even restack to form graphite (Figure 2f). Additionally, the SEM images of PTT-PTMO/ SWCNT+GNPs in Figures $2 \mathrm{~g}$ and $2 \mathrm{~h}$ show that the SWCNTs and graphene nanoplatelets form a kind of network structure. The SWCNTs appear to a have good affinity for GNPs. Aforementioned formation of nanofiller-nanofiller interactions may explain the good thermal stability and mechanical characteristics of PTT-PTMO/0.3 SWCNT+0.GNPs. The dispersion state in the composites with hybrid CNTs/ graphene particles was further confirmed by transmission microscopy.

The TEM images (Figure $3 \mathrm{c}$ and $3 \mathrm{~d}$, with two different magnifications of PTT-PTMO/0.1 SWCNTs+ 0.3 GNPs) display the real distribution of conductive fillers in the respective nanocomposites. They may provide valuable insight into the mechanisms behind the synergy between the hybrid fillers of CNT and graphene platelets in enhancing the conductivity [25] of nanocomposites. Figure 3c depicts both randomly dispersed individual SWCNTs and a round SWCNT agglomerate, also well-dispersed graphene sheets can be seen. In some places carbon nanotubes and graphene plates seem to be connected to one another. This can be better seen in Figure 3d where the dispersion of both nanofillers is even better and only nanotubes attached to graphene planes were observed.

In order to examine the dispersion of SWCNTs/ GNPs (with $0.3 \mathrm{wt} \%$ of SWCNTs and $0.1 \mathrm{wt} \%$ of GNPs) in the polymer hybrids in detail, TEM studies were carried out. They allow a qualitative understanding of the internal structure through direct observation. Typical TEM photographs of hybrids with $0.3 \mathrm{wt} \%$ of SWCNT and $0.1 \mathrm{wt} \%$ of GNPs are shown in Figure 3e and 3f. They show that both nanofillers were well dispersed in the polymer matrix, meaning that single nanotubes along with homogenously distributed GNPs were observed. Interestingly, the 'pull-out' mechanism of SWCNTs has been also shown as carbon nanotubes counteracted the break of the polymer matrix, which can clearly be seen at a higher magnification. As observed in the figures, the CNT-to-CNT and CNT-to-GNPs interactions by the surface contact (or tunnelling) should increase the mechanical strength of the composite, which will be discussed further.

\subsection{Phase structure}

An introduction of a carbon nanofiller into the multiphase system (block copolymer) may initiate the formation of a heterophase structure. The newly formed structure stabilizes the distribution of nanoparticles in the polymer matrix. The poly(ether-ester) multiblock copolymer composed of PTT as rigid segment and poly(tetramethylene oxide) PTMO as flexible segment with hardness of 51 Shore D [21, 23], was used as polymer matrix in nanocomposites described herein. During cooling from the melt, mentioned segmented block copolymer's microphase separated into high meting crystalline PTT hard domains and relatively low glass transition soft PTMO-rich domains. The elastic characteristics of this segmented block copolymer is a consequence of aforementioned microseparated phase structure, which is a result of the chemical nature and incompatibility between the rigid and flexible blocks/segments build into the polymer chains. Here, in order to investigate the effect of the addition of carbon nanostructures, which differ in shape, on the nanoseparated phase structure of the synthesized PTT-PTMO block copolymers, DSC and DMTA measurements were performed.

Forming of above mentioned separation occurs together with the formation of a soft (PTMO-rich) phase and a hard semicrystalline polyester (PTT) phase with characteristic temperatures $T_{\mathrm{g}}$ and $T_{\mathrm{m}}$, respectively. Existence of such a structure was confirmed by DSC measurement (Figure $4 a$ and $4 b$ ). Since the size of domains of the hard phase (few $\mathrm{nm},[20])$ is comparable to the size of SWCNTs and graphene nanoplatelets which were used to obtain the hybrid nanocomposites, it is difficult to explain the influence of hybrid nanofillers on the microstructure of the resulting nanocomposites. The glass transition temperatures $\left(T_{\mathrm{g} 1}\right.$ and $\left.T_{\mathrm{g} 2}\right)$ of nanocomposites were not affected by the incorporation of SWCNTs together with graphene nanoplatelets. Both nanofillers, despite good interactions with the polymer matrix (confirmed by SEM analysis), showed no effect on glass transition temperatures of both soft $\left(T_{\mathrm{g} 1}\right)$ and amorphous polyester phase $\left(T_{\mathrm{g} 2}\right)$.

In contrast, the crystallization process was stronger affected in the obtained nanocomposites. A notable rise in the crystallization temperature has been observed along with an increase in content of both 

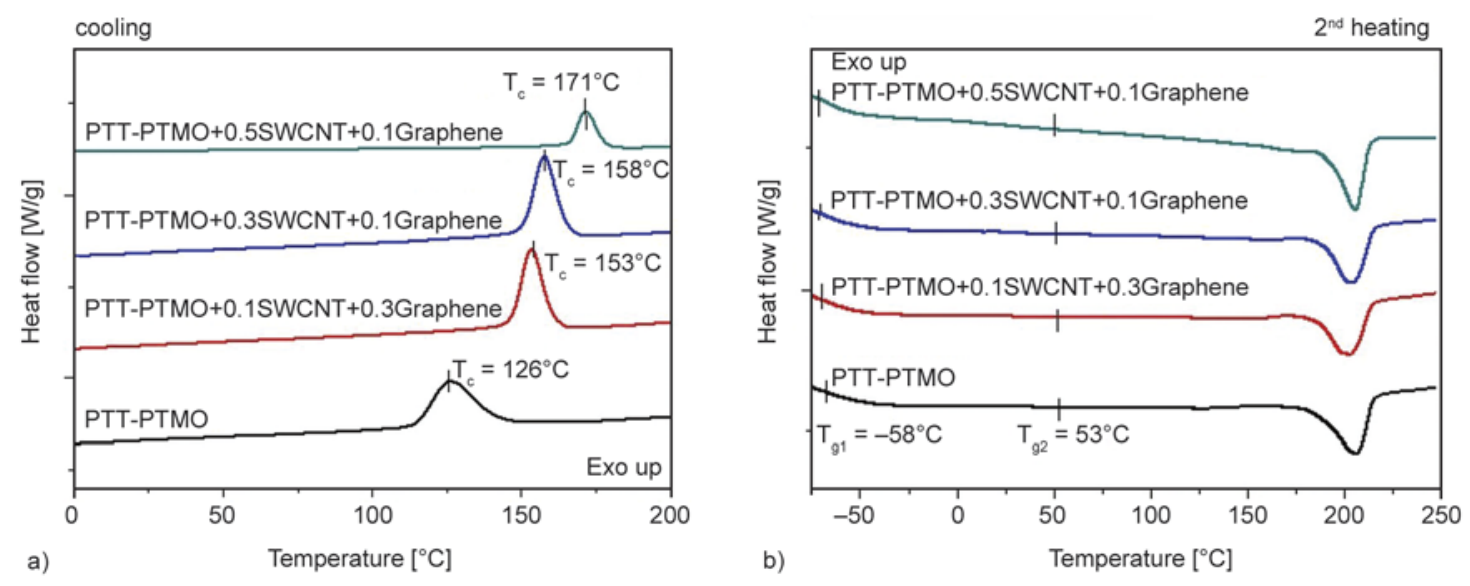

Figure 4. DSC thermograms for PTT-PTMO and PTT-PTMO/SWCNTs+GNPs nanocomposites during the cooling (a) and $2^{\text {nd }}$ heating (b)

SWCNTs and graphene nanoplatelets. The highest shift toward higher $T_{\mathrm{c}}$ temperature was observed in the case of PTT-PTMO/0.5 SWCNT+0.1 GNP, the value in question reached as high as $45^{\circ} \mathrm{C}$. Surprisingly, a stronger influence on crystallization behaviour seems to occur in the presence of carbon nanotubes in PTT-PTMO matrix. It can be well observed for hybrid nanocomposites where, with increasing loading of SWCNT (from 0.1 to 0.5 ), an increase in $T_{\mathrm{c}}$ was observed. However, all values of $\Delta H_{\mathrm{c}}$ were comparable to neat PTT-PTMO segmented block copolymer. In addition, the melting temperature remains unchanged for all nanocomposites. Based on the obtained results it can be also concluded that the composition of SWCNT:GNP of 5:1 accelerated the rate of crystallization probably due to larger amount of crystallization agents. It can clearly be seen that the $T_{\mathrm{c}}$ value increased along with an increase in SWCNTs content. The degree of crystallinity of obtained nanocomposites estimated basis DSC changes significantly (1-2\%) compared to neat block copolymer (Table 2). At first slight a decrease can be observed, but with increasing loading of both nanofillers a rise in comparison to neat PTT-PTMO emerges.

The DMTA analysis (Figure 5) has shown that PTTPTMO based nanocomposites are multiphase systems having one crystalline and two amorphous phases which confirmed the results obtained from DSC measurements. On the storage modulus $E^{\prime}$ curve at low temperatures an inflection (corresponding to the $\beta_{1}$ maximum in the loss modulus $E^{\prime \prime}$ ) associated with the glass transition of the soft polyether-rich phase can be observed. A decrease and a subsequent rise of the storage modulus value in the temperature range $20-120^{\circ} \mathrm{C}$ is related to amorphous PTT glass transition which is immediately followed by cold crystallization. (A completely amorphous PTT has a glass transition temperature of $40^{\circ} \mathrm{C}$, exceeding that value will cause its crystallization). Above $20^{\circ} \mathrm{C}$, the storage modulus depends on the type and content of carbon nanofiller. In the curve presenting the loss

Table 2. Thermal properties of neat PTT-PTMO and PTT-PTMO/SWCNTs+GNPs nanocomposites determined by DSC

\begin{tabular}{|l|c|c|c|c|c|c|c|c|c|}
\hline \multicolumn{1}{|c|}{ Sample } & $\begin{array}{c}\mathbf{T}_{\mathbf{g} 1} \\
{\left[{ }^{\circ} \mathbf{C}\right]}\end{array}$ & $\begin{array}{c}\mathbf{T}_{\mathbf{g} 2} \\
{\left[{ }^{\circ} \mathbf{C}\right]}\end{array}$ & $\begin{array}{c}\mathbf{T}_{\mathbf{m}} \\
{\left[{ }^{\circ} \mathbf{C}\right]}\end{array}$ & $\begin{array}{c}\Delta \mathbf{H}_{\mathbf{m}} \\
{[\mathbf{J} / \mathbf{g}]}\end{array}$ & $\begin{array}{c}\mathbf{T}_{\mathbf{c}} \\
{\left[{ }^{\circ} \mathbf{C}\right]}\end{array}$ & $\begin{array}{c}\Delta \mathbf{H}_{\mathbf{c}} \\
{[\mathbf{J} / \mathbf{g}]}\end{array}$ & $\begin{array}{c}\mathbf{x}_{\mathbf{c}} \\
{[\mathbf{\%}]}\end{array}$ & $\begin{array}{c}\mathbf{T}_{\boldsymbol{\beta} \mathbf{1}} \\
{\left[{ }^{\circ} \mathbf{C}\right]}\end{array}$ & $\begin{array}{c}\mathbf{T}_{\boldsymbol{\beta} \mathbf{2}} \\
{\left[{ }^{\circ} \mathbf{C}\right]}\end{array}$ \\
\hline PTT-PTMO & -58 & 53 & 206 & 30.9 & 126 & 28.3 & 21.2 & -31 & 43 \\
\hline PTT-PTMO/0.1 G & -61 & 52 & 204 & 28.1 & 149 & 27.3 & 19.2 & -35 & - \\
\hline PTT-PTMO/0.3 G & -59 & 50 & 205 & 29.1 & 149 & 27.8 & 19.9 & -36 & - \\
\hline PTT-PTMO/0.1 SWCNT+0.3G & -61 & 51 & 203 & 31.1 & 153 & 27.8 & 21.4 & -41 & 70 \\
\hline PTT-PTMO/0.3 SWCNT+0.1G & -62 & 51 & 203 & 31.7 & 158 & 28.8 & 21.7 & -41 & 75 \\
\hline PTT-PTMO/0.5 SWCNT+0.1G & -63 & 54 & 206 & 34.6 & 171 & 34.5 & 23.7 & -41 & 60 \\
\hline PTT-PTMO/0.1 SWCNT & -59 & 50 & 208 & 29.9 & 155 & 29.9 & 20.5 & -37 & 57 \\
\hline PTT-PTMO/0.3 SWCNT & -61 & 51 & 204 & 31.3 & 157 & 29.2 & 21.4 & -40 & 60 \\
\hline
\end{tabular}

$T_{\mathrm{g} 1}$ - glass transition temperature of soft phase, $T_{\mathrm{g} 2}$ - glass transition temperature of amorphous polyester phase, $T_{\mathrm{m}}-$ melting temperature of polyester crystalline phase; $T_{\mathrm{c}}$ - crystallization temperature of polyester crystalline phase; $\Delta H_{\mathrm{m}}, \Delta H_{\mathrm{c}}-$ enthalpy of melting and crystallization of polyester crystals, respectively; $x_{\mathrm{c}}$ - mass fraction of crystallinity, $T_{\beta 1}, T_{\beta 2}$ - temperature of $\beta_{1}$ - and $\beta_{2}-$ relaxations corresponding to the glass transition temperatures determined from maximum of $\tan \delta$ 

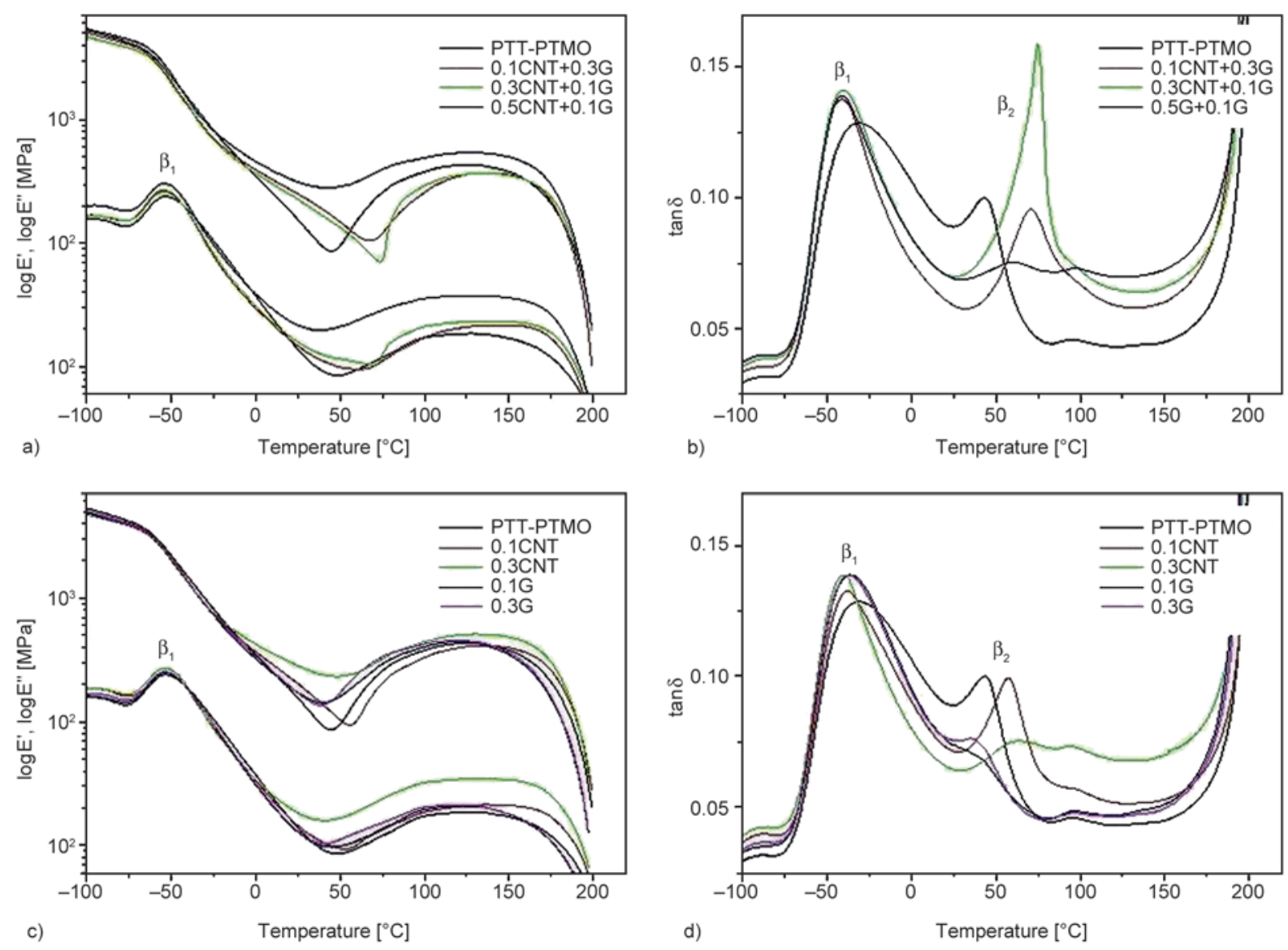

Figure 5. The dependence of storage modulus $E^{\prime}$, loss modulus $E^{\prime \prime}$ and $\tan \delta$ on temperature for PTT-PTMO/SWCNT $+\mathrm{G}$ (a)-(b) and PTT-PTMO/SWCNT and PTT-PTMO/G (c)-(d) nanocomposites

factor tangent of the temperature dependency (Figures $5 \mathrm{~b}, 5 \mathrm{c})$, two relaxations $\left(\beta_{1}, \beta_{2}\right)$ can clearly be seen. These relaxations are attributed to the glass transition of amorphous polyether-rich phase $\left(\beta_{1}\right)$ and amorphous polyester phase $\left(\beta_{2}\right)$. When a hybrid nanofiller in the form of a SWCNTs and GNPs mixture has been added (marked on the plot as $G$ ), a clear phase separation occurred. As a result two parts emerged, a PTMO-rich amorphous part (low temperature peak, $\beta_{1}$ ) and an amorphous PTT part (high temperature peak, $\beta_{2}$ ). Moreover, the $\beta_{1}$ peak has shifted towards lower temperatures and has become slimmer and narrower than the one corresponding to the neat PTT-PTMO, which proves that the presence of SWCNTs and GNPs influences the phase separation of the examined system. Also, the $\beta_{2}$ peak $(\max \tan \delta)$ has shifted towards higher temperatures and a change in its intensity can be observed, i.e. in the case of composites containing only graphene nanoplatelets $(0.1$ and 0.3$)$ or $(0.3 \mathrm{CNT}$, $0.5 \mathrm{CNT}+0.1 \mathrm{G})$ a decrease of $\beta_{2}$ peak's intensity can be seen, which is a clear evidence for a drop in PTT chain mobility.

\subsection{Thermal stability}

Thermogravimetric analysis (TGA) was used to analyze the effect of the hybrid system of nanofillers $(1 \mathrm{D}+2 \mathrm{D})$ dispersed in PTT-PTMO block copolymer matrix on its thermal and thermo-oxidative stability. The TGA curves for PTT-PTMO/ hybrid nanoparticles nanocomposites in air and an inert (argon) atmosphere are presented in Figure 6. No improvement in thermal stability has been observed in an inert atmosphere, as compared to the neat PTT-PTMO block copolymer, but also to PTTPTMO/SWCNTs and PTT-PTMO/GNPs nanocomposites (Table 3). What is more, a slight decrease in the temperatures of 5 and $10 \%$ weight loss $\left(T_{5 \%}\right.$, $\left.T_{10 \%}\right)$ was observed. However, in an oxidizing atmosphere for all hybrid nanocomposites the noticeable improvement of thermo-oxidative stability of nanofillers has been found. When comparing the temperature of 5\% weight loss for neat PTT-PTMO block copolymer and the hybrid composites, a significant shift of $24^{\circ} \mathrm{C}$ towards higher temperatures was observed. Interestingly enough, the addition of carbon nanofillers of different shapes and aspect ratios 

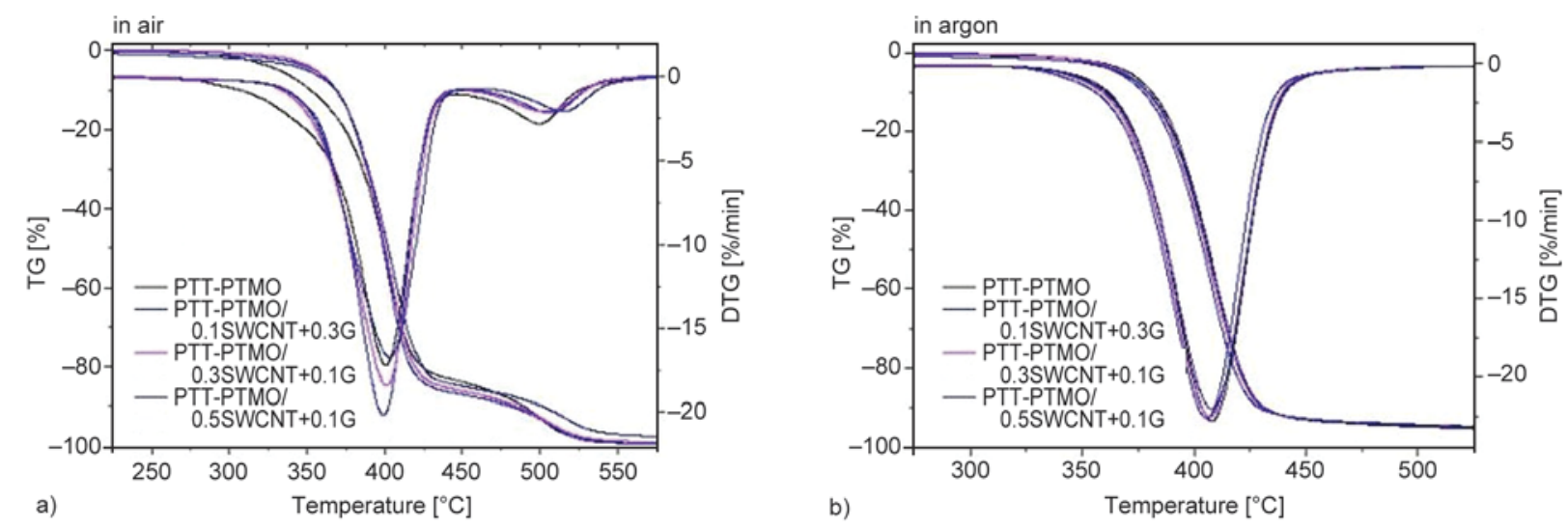

Figure 6. Weight loss and derivative weight loss versus temperature for the PTT-PTMO/SWCNTs+GNPs nanocomposites in air (a) and in argon (b) at a heating rate of $10^{\circ} \mathrm{C} / \mathrm{min}$

causes a substantial improvement in the thermooxidative stability of the thermoplastic elastomer in question. The thermal stability enhancement does not seem to depend upon a particular shape of the nanofiller as similar results were obtained in the case of SWCNTs and graphene nanosheets individually, as well as for the mixture of both. Apparently, both types of nanoparticles (carbon nanotubes and graphene nanoplatelets) affect the thermal stability of polymers in a similar manner. This can clearly be seen in Figure 6 where all the curves are close each other. Thus, it can be assumed that the morphology differences in PTT-PTMO/SWCNTs+GNPs nanocomposites do not appear to make a noticeable dif-

Table 3. Temperatures corresponding to 5 and $10 \%$ weight loss and the temperature at maximum of weight loss rate for the PTT-PTMO/ SWCNT+GNPs (G) nanocomposites obtained in air and argon atmosphere

\begin{tabular}{|c|c|c|c|c|}
\hline Symbol & $\begin{array}{l}\mathbf{T}_{5 \%} \\
{\left[{ }^{\circ} \mathbf{C}\right]}\end{array}$ & $\begin{array}{l}\mathbf{T}_{10 \%} \\
{\left[{ }^{\circ} \mathrm{C}\right]}\end{array}$ & $\begin{array}{c}\text { T }_{\text {DTG1 }} \\
{\left[{ }^{\circ} \mathbf{C}\right]}\end{array}$ & $\begin{array}{c}\text { T }_{\text {DTG2 }} \\
{\left[{ }^{\circ} \mathbf{C}\right]}\end{array}$ \\
\hline \multicolumn{5}{|c|}{ Measurement carried out in an oxidizing atmosphere } \\
\hline PTT-PTMO & 331 & 352 & 400 & 499 \\
\hline PTT-PTMO/0.1 G & 322 & 342 & 398 & 500 \\
\hline PTT-PTMO/0.3 G & 357 & 368 & 397 & 499 \\
\hline PTT-PTMO/0.1 SWCNT+0.1 G & 355 & 368 & 399 & 507 \\
\hline PTT-PTMO/0.3 SWCNT+0.3 G & 355 & 358 & 401 & 504 \\
\hline PTT-PTMO/0.5 SWCNT+0.3 G & 350 & 369 & 403 & 515 \\
\hline PTT-PTMO/0.1 SWCNT & 363 & 376 & 402 & 510 \\
\hline PTT-PTMO/0.3 SWCNT & 358 & 372 & 406 & 515 \\
\hline \multicolumn{5}{|c|}{ Measurement carried out in argon } \\
\hline PTT-PTMO & 371 & 382 & 408 & - \\
\hline PTT-PTMO/0.1 G & 366 & 383 & 406 & - \\
\hline PTT-PTMO/0.3 G & 374 & 382 & 408 & - \\
\hline PTT-PTMO/0.1 SWCNT+0.1 G & 366 & 377 & 406 & - \\
\hline PTT-PTMO/0.3 SWCNT+0.3 G & 368 & 379 & 407 & - \\
\hline PTT-PTMO/0.5 SWCNT+0.3 G & 367 & 380 & 409 & - \\
\hline PTT-PTMO/0.1 SWCNT & 372 & 382 & 408 & - \\
\hline PTT-PTMO/0.3 SWCNT & 369 & 380 & 408 & - \\
\hline
\end{tabular}

ference in the thermal stability of the nanocomposites. The possible explanation for such behaviour is that both the SWCNTs and graphene nanoplatelets have high electron affinities and as such seem to be capturing free radicals [30, 31]. Discussed TGA results suggest that by incorporating either SWCNTs or graphene nanoplatelets or the mixture of both into the polymer, its thermo-oxidative stability should be improved.

\subsection{Mechanical properties}

The use of new carbon nanomaterials with excellent mechanical properties has opened a new and exciting chapter in today's material science. A better understanding of chemistry and the intrinsic properties of both graphene and carbon nanotubes, along with new and innovative approaches to polymer fabrication has led scientists to developing composites with GNPs and SWCNTs and thus creating new materials with substantially enhanced mechanical, electrical, thermal and barrier properties. Similarly to traditional composites, the range of the obtained improvements is referred to using factors such as reinforcement phase concentration, distribution in the host matrix, interface bonding and the reinforcement phase aspect ratio.

The tensile properties of the PTT-PTMO/SWCNTs + GNPs hybrid nanocomposites were examined. Typical stress-strain curves for PTT-PTMO block copolymers were plotted in Figure 7. In order to make the plots more comprehensible, the obtained data is presented in two separate figures where the results obtained for hybrid nanocomposites have been compared to those for graphene (a) and carbon nanotubes (b). A clear tendency for an increase in tensile modulus and a decrease in elongation, caused by a 

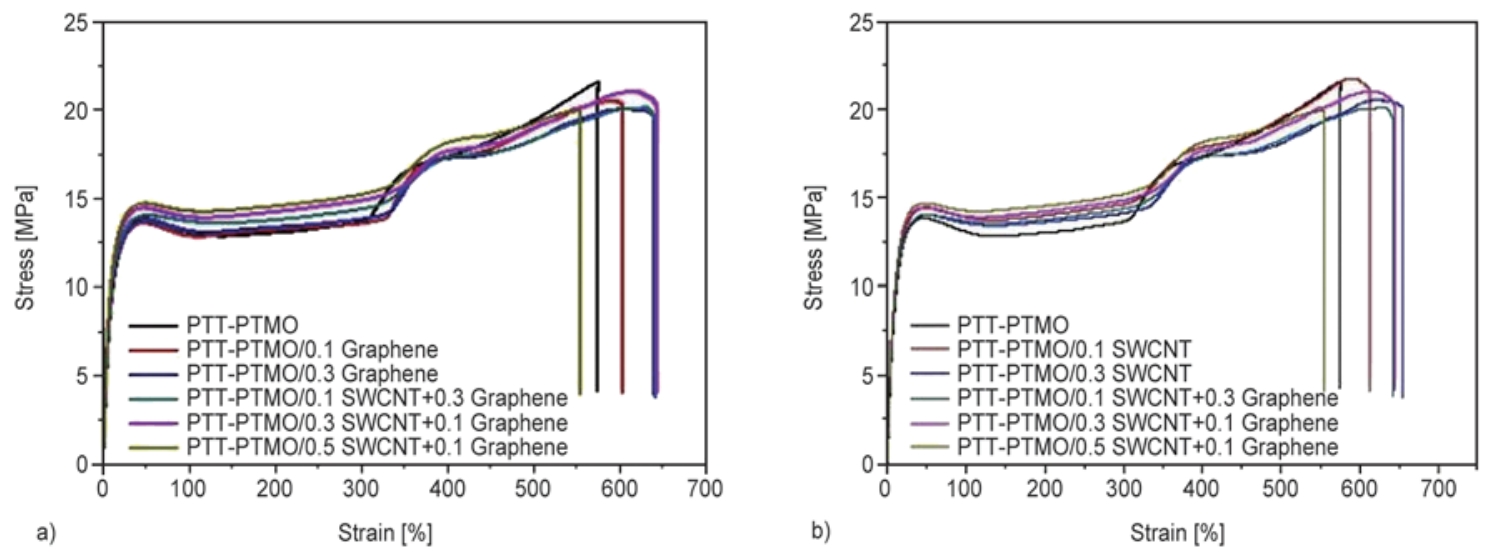

Figure 7. Representative stress-strain curves of PTT-PTMO/SWCNTs+GNPs hybrid nanocomposites (a) in comparison to PTT-PTMO/GNPs and (b) in comparison to PTT-PTMO/SWCNTs nanocomposites

rise in the hybrid system content has been observed (Table 4). Carbon nanofillers (SWCNTs and GNPs) added in small amounts to PTT-PTMO elastomer increase the tension related to deformation, limiting the free length of chains located between physical nodes of the network/matrix (they create additional physical nodes). This effect was significantly stronger when two nanofillers were used. For pristine PTTPTMO the tensile modulus was $118 \mathrm{MPa}$. With the incorporation of up to $0.3 \mathrm{wt} \%$ of graphene, the tensile modulus decreased to ca. $131 \mathrm{MPa}$, which is ca. $11 \%$ more compared to neat PTT-PTMO block copolymer. However, in the case of PTT-PTMO/ SWCNTs a substantial rise up to $162 \mathrm{MPa}$ has been noted. The increase in the modulus was accompanied by lower values of elongation at break for nanocomposites with graphene and with $0.1 \mathrm{wt} \%$ of SWCNTs. Only a slight increase in $\varepsilon b$ for PTTPTMO/0.3 SWCNT to $639 \%$ was observed. An addition of the hybrid system has further increased the modulus to 149,178 and $212 \mathrm{MPa}$, for 0.1 SWCNTs + $0.3 \mathrm{G}, 0.3 \mathrm{SWCNTs}+0.1 \mathrm{GNPs}$ and $0.5 \mathrm{SWCNTs}+$ 0.1 GNPs respectively. Hybrid nanocomposite with a concentration of $0.5 \mathrm{wt} \%$ of nanotubes and $0.1 \mathrm{wt} \%$ of graphene reached a modulus value which is ca. $68 \%$ higher than the pristine elastomer. Thus, the tensile measurements revealed that the effect of hybrid nanofillers system is more pronounced on the tensile modulus. The noticeable increase in the tensile modulus reflects the reinforcement effect attained by the dispersion of the nanotubes and graphene platelets in PTT-PTMO matrix. Calorimetric studies for obtained PTT-PTMO/hybrid nanocomposites (Table 4) showed only minute differences in the polymer melt enthalpy and negligible increase in degree of crystallinity. Therefore, the improvement in the tensile properties at low nanofillers' loading observed herein, cannot be due to a change in crystallinity and is more likely caused by the presence of SWCNTs and graphene nanoplatelets next to PTT hard domains dispersed in PTMO-rich soft phase. Similar results have been also previously observed for PTT-PTMO nanocomposites with graphene oxide (GO) [24] and montmorillonite (MMT) [22].

Elongation at break, on the other hand, decreased significantly for PTT-PTMO/0.5 SWCNTs+0.1 GNPs. At the same time, a rise in the permanent set accom-

Table 4. Tensile properties of PTT-PTMO/SWCNTs+GNPs nanocomposites

\begin{tabular}{|l|c|c|c|c|c|c|}
\hline \multicolumn{1}{|c|}{ Sample } & $\begin{array}{c}\mathbf{E} \\
{[\mathbf{M P a}]}\end{array}$ & $\begin{array}{c}\boldsymbol{\sigma} \mathbf{( 1 0 0 \% )} \\
{[\mathbf{M P a}]}\end{array}$ & $\begin{array}{c}\mathbf{\sigma}_{\mathbf{y}} \\
{[\mathbf{M P a}]}\end{array}$ & $\begin{array}{c}\mathbf{\varepsilon}_{\mathbf{y}} \\
{[\mathbf{\%}]}\end{array}$ & $\begin{array}{c}\mathbf{\sigma}_{\mathbf{b}} \\
{[\mathbf{M P a}]}\end{array}$ & $\begin{array}{c}\mathbf{\varepsilon}_{\mathbf{b}} \\
{[\mathbf{\%}]}\end{array}$ \\
\hline PTT-PTMO & $118.3 \pm 1.5$ & $12.7 \pm 0.1$ & $13.6 \pm 0.2$ & $44.8 \pm 0.9$ & $20.3 \pm 0.7$ & $625 \pm 12$ \\
\hline PTT-PTMO/0.1 GNPs & $123.0 \pm 1.3$ & $12.8 \pm 0.1$ & $13.4 \pm 0.2$ & $50.4 \pm 1.3$ & $20.3 \pm 0.1$ & $594 \pm 12$ \\
\hline PTT-PTMO/0.3 GNPs & $131.7 \pm 4.1$ & $13.1 \pm 0.1$ & $13.7 \pm 0.1$ & $49.6 \pm 0.1$ & $19.8 \pm 0.2$ & $594 \pm 14$ \\
\hline PTT-PTMO/0.1 SWCNT+0.3 GNPs & $149.2 \pm 2.7$ & $13.6 \pm 0.1$ & $14.0 \pm 0.1$ & $49.8 \pm 0.8$ & $19.2 \pm 0.3$ & $634 \pm 29$ \\
\hline PTT-PTMO/0.3 SWCNT+0.1 GNPs & $178.3 \pm 6.9$ & $13.9 \pm 0.1$ & $14.4 \pm 0.1$ & $48.1 \pm 1.1$ & $20.4 \pm 0.2$ & $642 \pm 21$ \\
\hline PTT-PTMO/0.5 SWCNT+0.1 GNPs & $212.2 \pm 6.5$ & $14.1 \pm 0.1$ & $14.6 \pm 0.9$ & $47.6 \pm 1.2$ & $18.5 \pm 1.2$ & $542 \pm 59$ \\
\hline PTT-PTMO/0.1 SWCNT & $145.1 \pm 3.9$ & $13.6 \pm 0.3$ & $14.2 \pm 0.2$ & $47.8 \pm 0.8$ & $20.7 \pm 0.6$ & $617 \pm 24$ \\
\hline PTT-PTMO/0.3 SWCNT & $162.5 \pm 2.5$ & $13.7 \pm 0.1$ & $14.1 \pm 0.1$ & $49.7 \pm 0.5$ & $19.9 \pm 0.2$ & $639 \pm 13$ \\
\hline
\end{tabular}

$E$ - tensile modulus; $\sigma(100 \%)$ - stress at strain of $100 \% ; \sigma_{\mathrm{y}}, \varepsilon_{\mathrm{y}}-$ yield stress and strain respectively, $\sigma_{\mathrm{b}}, \varepsilon_{\mathrm{b}}-$ stress and strain at break respectively 


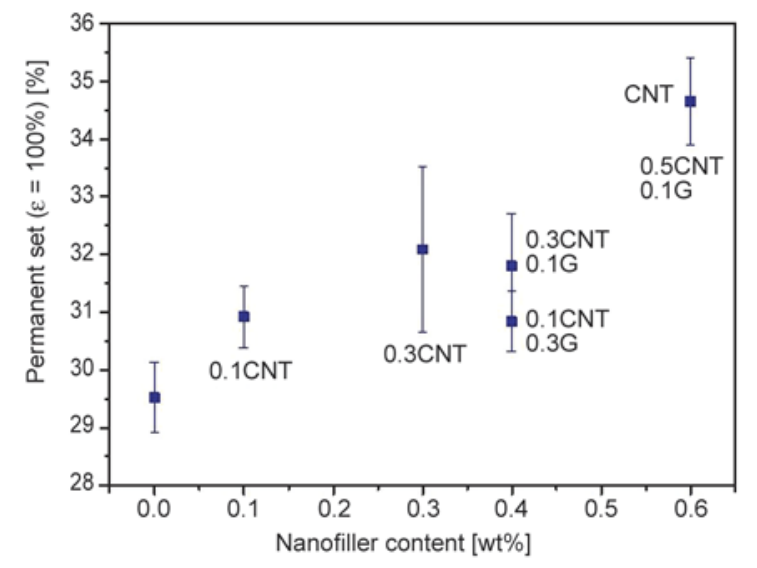

Figure 8. Permanent set accompanying the $100 \%$ deformation as a function of total content of carbon nanofillers for nanocomposites based on PTT-PTMO

panying the $100 \%$ deformation was observed in a limited reversible deformation range. Analysis of the permanent set, created after a $100 \%$ deflection for the examined PTT-PTMO composites (Figure 8) proved that content of nanofillers in the amount of up to $0.6 \mathrm{wt} \%$ cause only a slight deterioration of elasticity as they cause just a few percent decrease in relaxation from $100 \%$ deflection. It can be said however, that nanofillers greatly impede the elasticity, because at $0.6 \mathrm{wt} \%$ content an average of the permanent set increased by $20 \%$. The synergistic toughening mechanisms of a combination of SWCNTs and graphene has been observed for PTT-PTMO block copolymer.

\section{Conclusions}

The present research and experiments focused mainly on the preparation and characterization of polymer composites containing SWCNTs, GNPs and hybrid systems comprising of both SWCNTs/ GNPs, in which PTT-PTMO constituted the matrix. The use of in situ synthesis allowed to obtain composites with a high degree of homogeneity, which is a key issue for further industrial applications. At the same time, the analysis of the properties of obtained materials showed a strong effect of the addition of carbon nanotubes and graphene nanoplatelets on their structure, physical transitions, thermal stability and mechanical strength. The presence of SWCNTs and GNPs in the polymer melt during cooling substantially speeds up the crystallization process as is evidenced by a shift of the crystallization peak to up to $45^{\circ} \mathrm{C}$, which was recorded for PTT-PTMO/ $0.5 \mathrm{SWCNT}+0.1 \mathrm{GNP}$ hybrid nanocomposite. The introduction of carbon nanoparticles into the poly- mer matrix did not have a significant effect on the thermal stability of obtained nanocomposites in an inert atmosphere. However, addition of SWCNTs and graphene nanoplatelets individually, as well as the mixture of both caused substantial enhancement of thermo-oxidative stability, shifting the beginning of the chemical decomposition temperature by 20 $25^{\circ} \mathrm{C}$. Remarkably, in the case of mechanical properties of presented nanocomposites a synergistic hybrid effect has been observed. The addition of carbon nanotubes together with graphene nanosheets caused an increase in strength of up to $68 \%$ for the highest concentration of nanofillers. Results of the experiments contained herein indicate a remarkable potential of the new group of materials, the polymer hybrid nanocomposites, which and might be of extreme importance for further development and possible industrial applications. Determination of the appropriate conditions for obtaining hybrid nanocomposites during the polymer matrix synthesis process is paramount in order to develop new technologies for their production on the pilot and industrial scale. Moreover, the observed positive hybrid effect in the case of segmented block copolymers will allow to lower the final price of the finished product (suitable selection of properties at the optimum price), which can be characterized by good thermal properties with, at the same time, improved mechanical properties.

\section{Acknowledgements}

This work was financially supported by National Science Center in Poland, project Preludium 2013/11/N/ST8/00404. Authors would like to thank Dr Ronit Popovitz for preparing TEM micrographs.

\section{References}

[1] Thomas S., Zaikow G. E.: Progress in polymer nanocomposite research. Nova Science, New York (2008).

[2] Wang Z. M.: Toward functional nanomaterials. Springer, New York (2009).

[3] Kingston C., Zepp R., Andrady A., Boverhof A., Fehir R., Hawkins D., Roberts J., Sayre P., Shelton B., Sultan Y., Vejins V., Wohlleben W.: Release characteristics of selected carbon nanotube polymer composites. Carbon, 1, 33-57 (2014).

DOI: $10.1016 /$ j.carbon.2013.11.042

[4] Kim H., Abdala A. A., Macosko C. W.: Graphene/polymer nanocomposites. Macromolecules, 43, 6515-6530 (2010).

DOI: $10.1021 / \mathrm{ma1} 00572 \mathrm{e}$ 
[5] Slonczewski J. C., Weiss P. R.: Band structure of graphite. Physical Review Letters, 109, 272-279 (1958). DOI: 10.1103/PhysRev.109.272

[6] Soldano C., Mahmood A., Dujardin E.: Production, properties and potential of graphene. Carbon, 48, 21272150 (2010).

DOI: $10.1016 /$ j.carbon.2010.01.058

[7] Novoselov K. S., Geim A. K., Morozov S. V., Jiang D., Zhang Y., Dubonos S. V., Grigorieva I. V., Firsov A. A.: Electric field effect in atomically thin carbon films. Science, 306, 666-669 (2004). DOI: $10.1126 /$ science. 1102896

[8] Lee C., Wei X., Kysar J. W., Hone J.: Measurement of the elastic properties and intrinsic strength of monolayer graphene. Science, 321, 385-388 (2008). DOI: $10.1126 /$ science.1157996

[9] Balandin A. A., Ghosh S., Bao W., Calizo I., Teweldebrhan D., Miao F., Lau C. N.: Superior thermal conductivity of single-layer graphene. Nano Letters, 8, 902907 (2008).

DOI: $10.1021 / \mathrm{n} 10731872$

[10] Bunch J. S., Verbridge S. S., Alden J. S ., van der Zande A. M., Parpia J. M., Craighead H. G., McEuen P. L.: Impermeable atomic membranes from graphene sheets. Nano Letters, 8, 2458-2462 (2008).

DOI: $10.1021 / \mathrm{nl} 801457 \mathrm{~b}$

[11] Kim H., Miura Y., Macosko C. W.: Graphene/polyurethane nanocomposites for improved gas barrier and electrical conductivity. Chemistry of Materials, 22, 34413450 (2010).

DOI: $10.1021 / \mathrm{cm} 100477 \mathrm{v}$

[12] El Achaby M., Arrakhiz F-A., Vaudreuil S., el Kacem Qaiss A., Bousmina M., Fassi-Fehri O.: Mechanical, thermal, and rheological properties of graphene-based polypropylene nanocomposites prepared by melt mixing. Polymer Composites, 33, 733-744 (2012).

DOI: $10.1002 /$ pc. 22198

[13] Hall L. J., Coluci V. R., Galvão D. S., Kozlov M. E., Zhang M., Dantas S. O., Baughman R. H.: Sign change of Poisson's ratio for carbon nanotube sheets. Science, 320, 504-507 (2008).

DOI: $10.1126 /$ science. 1149815

[14] Park J. G., Li S., Liang R., Fan X., Zhang C., Wang B.: The high current-carrying capacity of various carbon nanotube-based buckypapers. Nanotechnology, 19, 185710/1-185710/7 (2008).

DOI: $10.1088 / 0957-4484 / 19 / 18 / 185710$

[15] Cai D., Song M., Xu C.: Highly conductive carbonnanotube/graphite-oxide hybrid films. Advanced Materials, 20, 1706-1709 (2008).

DOI: $10.1002 / \mathrm{adma} 200702602$

[16] Khan U., O’Connor I., Gun'ko Y. K., Coleman J. N.: The preparation of hybrid films of carbon nanotubes and nano-graphite/graphene with excellent mechanical and electrical properties. Carbon, 48, 2825-2830 (2010). DOI: $10.1016 /$ j.carbon.2010.04.014
[17] Yang S-Y., Lin W-N., Huang Y-L., Tien H-W., Wang JY., Ma C-C. M., Li S-M., Wang Y-S.: Synergetic effects of graphene platelets and carbon nanotubes on the mechanical and thermal properties of epoxy composites. Carbon, 49, 793-803 (2011). DOI: $10.1016 /$ j.carbon.2010.10.014

[18] Gabriëlse W., Soliman M., Dijkstra K.: Microstructure and phase behavior of block copoly(ether ester) thermoplastic elastomers. Macromolecules, 34, 1685-1693 (2001). DOI: $10.1021 / \mathrm{ma} 0012696$

[19] Schmalz H., Abetz V., Lange R., Soliman M.: New thermoplastic elastomers by incorporation of nonpolar soft segments in PBT-based copolyesters. Macromolecules, 34, 795-800 (2001). DOI: $10.1021 / \mathrm{ma} 001226 \mathrm{p}$

[20] Szymczyk A., Senderek E., Nastalczyk J., Roslaniec Z.: New multiblock poly(ether-ester)s based on poly(trimethylene terephthalate) as rigid segments. European Polymer Journal, 44, 436-443 (2008).

DOI: $10.1016 /$ j.eurpolymj.2007.11.005

[21] Szymczyk A., Nastalczyk J., Sablong R. J., Roslaniec Z.: The influence of soft segment length on structure and properties of poly(trimethylene terephthalate)-blockpoly(tetramethylene oxide) segmented random copolymers. Polymers for Advanced Technologies, 22, 72-83 (2011). DOI: $10.1002 /$ pat. 1858

[22] Szymczyk A., Paszkiewicz S., Roslaniec Z.: Influence of intercalated organoclay on the phase structure and physical properties of PTT-PTMO block copolymers. Polymer Bulletin, 70, 1575-1590 (2013).

DOI: 10.1007/s00289-012-0859-y

[23] Szymczyk A.: Poly(trimethylene terephthalate-blocktetramethylene oxide) elastomer/single-walled carbon nanotubes nanocomposites: Synthesis, structure, and properties. Journal of Applied Polymer Science, 126, 796-807 (2012). DOI: $10.1002 / a p p .36961$

[24] Paszkiewicz S., Szymczyk A., Špitalski Z., Mosnáček J., Kwiatkowski K., Rosłaniec Z.: Structure and properties of nanocomposites based on PTT-block-PTMO copolymer and graphene oxide prepared by in situ polymerization. European Polymer Journal, 50, 69-77 (2014).

DOI: 10.1016/j.eurpolymj.2013.10.031

[25] Paszkiewicz S.: Polymer hybrid nanocomposites containing carbon nanoparticles. In situ synthesis and physical properties. PhD thesis, West Pomeranian University of Technology in Szczecin, Institute of Materials Science and Engineering, Poland (2014).

[26] Paszkiewicz S., Rosłaniec Z., Szymczyk A., Spitalsky Z., Mosnácek J.: Morphology and thermal properties of expanded graphite (EG)/Poly(ethylene terephthalate) (PET) nanocomposites. Chemik, 66, 21-30 (2012). 
[27] Paszkiewicz S., Szymczyk A., Špitalský Z., Soccio M., Mosnáček J., Ezquerra T. A., Rosłaniec Z.: Electrical conductivity of poly(ethylene terephthalate)/expanded graphite nanocomposites prepared by in situ polymerization. Journal of Polymer Science Part B: Polymer Physics, 50, 1645-1652 (2012).

DOI: $10.1002 /$ polb. 23176

[28] Scheirs J., Long T. E.: Modern polyesters. Chemistry and technology of polyesters and copolyesters. Wiley, Chichester (2003).

[29] Pötschke P., Arnaldo M. H., Radusch H-J.: Percolation behavior and mehanical properties of polycarbonate composites filled with carbon black/carbon nanotube systems. Polimery, 3, 204-211 (2012).

DOI: $10.14314 /$ polimery.2012.204
[30] Yu J., Huang X., Wu C., Jiang P.: Permittivity, thermal conductivity and thermal stability of poly(vinylidene fluoride)/graphene nanocomposites. IEEE Transactions on Dielectrics and Electrical Insulation, 18, 478-484 (2011).

DOI: 10.1109/TDEI.2011.5739452

[31] Kuan C-F., Chen W-J., Li Y-L., Chen C-H., Kuan H-C., Chiang C-L.: Flame retardance and thermal stability of carbon nanotube epoxy composite prepared from solgel method. Journal of Physics and Chemistry of Solids, 71, 539-543 (2010).

DOI: $10.1016 /$ j.jpcs.2009.12.031 